\section{NOTES SUR LES COUPS DE BÉLIER}

de M.J. Boussinesq, à l'Académie des Sciences

I

Propagation des ondes le long d'une colonne liquide compressible, se composant de filets à vitesses inégales et remplissant un tuyau élastique horirontal, sans tension longrtudunale. $\left(^{*}\right)$

I. Résal me paraît avoir, le premier, ou un des premiers, dans une courte Note du 27 mars 1876 , soumis au calcul les mouvements que propage le long d'une colonne jiquide l'élasticité du tuyau qui la contient, comme sont, par exemple, les battements du pouls dans les artères, les ondes longitudinales de l'eau remplissant le tube en caoutchouc de certains appareils de Marey pour l'enregistrement ou l'imitation de phénoménes physiologiques, enfin, les coups de bélier provoqués, dans les tuyaux de conduite, par toute brusque variation de la vitesse d'écoulement et, par suite, de la pression. Comme Résal avait spécialement en vue la colonne liquide, sans écoulement, contenue dans un tube en caoutchouc généralement un peu plus long que la distance de ses deux extrémités et, d'ailleurs, incomparable. ment plus dilatable par la pression intérieure que n'est compressible le liquide, il a pu négliger, avec une certaine approximation, les actions mutuelles des anneaux juxtaposés composant le tube, et admettre le parallélisme des tranches avec l'incompressibililé des volumes fluides. Il obtient ainsi, pour le carré de la vitesse de propagation des ondes, le quotient, par la densité $p$ du liquide, de l'élasticité $E$ de traction du tube, multipliée par le petit rapport de l'épaisseur $\approx$ de la paroi au diamètre intérieur $2 R$.

Dans un Mémoire intitulé Théorie générale du mounement varié de l'eau dans les tuyaux de conduite (Remue de Mécanique, janvier et mars 1904), M. Alliévi a généralisé très heureusement, presque sans la compliquer, la formule de Résal, en tenant compte de la compressibilité du liquide, comme il le fallait bien, eu égard à la grande rigidité des tuyaux de conduite, mais en faisant encore l'hypothèse du parallélisme des tranches, rendue peu acceptable par l'inégale rapidité des filets fluides dans ces tuyaux, et en continuant enfin à admettre l'indépendance relative des anneaux circulaires contigus du tuyau.

Cette dernière hypothèse, quoique moins approchée pour un tel tuyau que pour un tube lâche, et quı ne serait pleinement justifiée que dans le cas d'une paroi non isotrope très-extensible suivant la longueur, est inévitable dès qu'on veut pouvoir traiter la question. Car le problème ne serait plus réductible à une seule équation aux dérivées partiellès, s'il fallait tenir compte des actions mutuelles des anneaux $\left.{ }^{* *}\right)$. Mais il y a lieu de ne pas négliger les inégalités de vitesse des filets fluides: c'est ce que je me propose de faire ici.

\section{Supposons d'abord en repos (comme le fait Résal), et}

(*) Séance du 3 juillet 1905 .

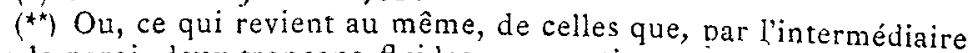
de la paroi, deux tronçons fluides non contigus pourratent exercer

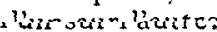

même sans pression, la colonne liquide, d'ailleurs dépour. vue de pesanteur ou horizontale, et à sections circulaires d'un rayon $R$ constant alitour de l'axe des $x$. Puis exerçons sur elle une pression uniforme qui lui fera éprouver, à partir d'une certaine section $x=0$, censée ètre restée dans son plan primitif, les petites contractions statiques, soit cubique, soit surtout en longueur, nécessaires à l'existence de cette pression, vu les légères dilatations latérales simultanées qui tendront uniformément les fibres annulaires du tuyau pour leur permettre d'équilibrer cette tension intérieure. Produi. sons enfin, à partir d'un moment donné $t=0$, sur le fluide ainsi revenu au repos, des variations de pression communes à toute la section $x=0$, en déplaçant, par exemple, celle-ci normalement à son plan.

Il en résultera plus ou moins vite, dans toute la colonne, des déplacements presque parallèles à l'axe et aussi, par suite, des variations de la pression $p$ sensiblement pareilles sur toute l'étendue des sections normales, ou fonction seulement de $x$ et de $t$. Chaque tronçon de la colonne, primitivement compris entre les abscisses $x_{0}$ et $x_{0}+\mathrm{d} x_{0}$ acquerra suivant les $x$, par l'effet des chutes de pression s'y observant, des vitesses longitudinales $u$ communes, assez lentement variables avec $x_{0}$ en raison de leur rapide propagation; et les tronçons se conserveront ainsi presque cylindriques durant des temps notables, à cause de la petitesse qu'ont les frottements dans les fluides.

Appelons $\xi$ le déplacement total. iusqu'à l'époque $t$ et suivant les $x$ de la première base du tronçon, d'abscisse primitive $x_{0}$, mais d'abscisse actuelle $x=x_{0}+\xi$; et soient o le petit écartement relatif $\frac{\mathrm{d}}{\mathrm{d} x_{0}}$ des deux bases du tronçon, $\partial$ 'la dilatation analogue, comparable à $\mathfrak{a}$, des rayons primitifs $R$ de celles-ci ou des fibres longitudinales $2 \pi R$ de l'anneau de paroi entourant le tronçon, fibres dont $E$ désignera le coelficient ordinaire d'élasticité; enfin, ミl'épaisseur du tuyau à l'état naturel, supposé petite devant $R$. L'accroissement $R \partial^{\prime}$ des rayons sera négligeable à côté de $\xi$; et, par suite, les vitesses et accélérations, suivant les rayons, tant du troncon fluide que de la paroi, seront peu de chose à côté de celles du mouvement longitudinal du fluide. C'est dire que les inerties en jeu dans le tuyau, transversales, ou même, par suite, longitudinales, et aussi celles du fluide suivant les sens normaux à l'axe, seront insensibles comparativement aux inerties du fluide suivant l'axe.

III. Or celles-ci, dues à la différence des pressions exercées sur les deux bases du tronçon, pressions presque égales, même quand la distance de ces bases est prise comparable à $R$, sont très faibles a côté de la pression sur une seule base et, par suite, à côté de la pression $p\left({ }_{2} R \mathrm{~d} x\right)$ sur une section méridienne $2 R \mathrm{~d} x$ du tronçon menée suivant l'axe, ou, encore, à côté de la tension, $\left(E \partial^{\prime}\right)(2 \leftleftarrows \mathrm{d} x)$ très-sensiblement, du demi-anneau de paroi limité par cette section méridienne, tension censée équilibrer la pression $2 R p \mathrm{~d} x$, aux inerties transversales près $\left(^{*}\right)$. Donc, à bien plus forte raison, les inerties transversales, tant du

(*) Puisque l'on néglige les actions mutuelles des anneaux du tuyau contigus, chaque anneau se comporte comme s'il était seul en présence du tronçon fluide sous-jacent de. même longueur. 
fuide que du tuyau, dont il s'agit ici, sont négligeables devant la pression $2 R p \mathrm{~d} x$; et il vient, par la suppression du facteur commun $2 \mathrm{~d} x$,

$$
E_{\varepsilon} \partial^{\prime}=R p ; \quad \text { d'où } \quad \partial^{\prime}=\frac{R}{\varepsilon} \frac{p}{E}
$$

D'autre part, si $k$ désigne le coetficient d'élasticité du fluide (inverse de la compressibilité), rapport de la pression $p$ à la contraction cubique $-\partial-2 d$, on a $-p=$ $k\left(\partial+2 \partial^{\prime}\right)$, formule d'où l'élımination de $\partial^{\prime}$ par (1) déduira la relation, caractéristifue du probleme, existant entre la tension - $p$ de la colonne par unité de section normale et l'allongenent relatif correspondant a. Appelant po la densité du liquide à l'état naturel, très peu différente de la densité effective $\rho$ sous la pression variable $p$, posons

$$
\frac{1}{\omega^{2}}=\frac{P_{0}}{k}+\frac{p_{0}}{E} \frac{2 R}{\varepsilon}
$$

et la formule caractéristique obtenue sera

$$
-p=\rho_{0} \omega^{2} \mathrm{a}=\rho_{0} \omega^{2} \frac{\mathrm{d} \xi}{\mathrm{d} x_{0}} .
$$

La méthode ordinaire pour le calcul de tous les petits mouvements longitudinaux en déduit immédiatement P'équation aux dérivées partielles du problème:

$$
\text { d'où, aussi, } \quad \frac{\mathrm{d}^{2}(p, U)}{\mathrm{d} t^{2}}=\omega^{2} \frac{\mathrm{d}^{2}(p, U)}{\mathrm{d} x_{0}^{2}} \text {, }
$$$$
\frac{\mathrm{d}^{2}=}{\mathrm{d} t^{2}}=\omega^{2} \frac{\mathrm{d}^{2} \%}{\mathrm{~d} x_{0}^{2}}
$$

Udésignarıt la vitesse moyenne de débit à travers chaque section, vitesse identique, ici, à la dérivée, $u$, de $\xi$ en $t$. Enfin, à raison de la petitesse de la dérivée de $\xi$ en $x_{0}$, l'on peut, sans changement appréciable des dérivées partielles de $\xi$, substituer à $t$ et à $x_{0}$, comme variables indépendantes, $t$ et $x_{0}+\xi$, c'est-à-dire $t$ et $x$; ce qui donne les équations définitives

$$
\frac{\mathrm{d}^{2}(p, U)}{\mathrm{d} l^{2}}=\omega^{2} \frac{\mathrm{d}^{2}(p, U)}{\mathrm{d} x^{2}}
$$

Ce sont celles de M. Alliévi, avec la valeur de la cilérité ou ritesse de propagation $\omega$ qui résulte de (2).

IV. Mais reconnaissons qu'elles subsistent, quand la colonne liquide est déjà, au moment où d'àssez rapides changements de la pression l'atteignent près d'une section $x=0$, en train de couler par filets rectilignes et parallèles inégalement rapides, animés de vitesse $u_{0}$ comparables à celles que vont produire ces changements et, par conséquen ${ }^{2}$ toujours très petites à côté de la célérité w. C'est ce qui arrive, par exemple, quand la longueur du tuyau" est sulfisante pour que les petits frottements des filets et de la paroi, quoique négligeables sur des parcours $x$ comme ceux que nous considérons ici, aient établi, concurremment avec une petite pente motirice ainsi neutralisée par eux, un régime uniforme dans la région des $x$ positifs.

Alors la première équation d'Euler, $u^{\prime}=-\frac{\mathrm{J}}{\mathrm{p}} \frac{\mathrm{d} p}{\mathrm{~d} x}$, est applicạble aux mouvements ondulatoires survenus assez vite; car les frottements et la petite composante de la pcsanteur suivant les $x$ (ou le petit décroissement analogue de la pression) y sont relativement insensibles. Or, les vitesses engendrées $u-u_{0}$ étant encore censées principalement longitudinales, la pression $p$ et, par suite, la densité p, continuent à ne dépendre guère que de $x$ et de $t$. Donc l'accélération $u$ est encore commune à toute une section $\sigma$ et même (vu la rapidıté de la propagation comparatwement à la difference des parcours effectifs jusqu à l'instant $t$ ), commune à tout le fluide d'une région de longueur modérée. Les accroissements $u-u_{0}$ de vitesse sont, par suite, pareils pour tout ce fluide et égaux à leur morenne, $U-U_{0}$ (a très peu près), dont la dérivée en $t$, prise sur place, exprime, dès lors, sensiblement $u^{\prime}$. Ainsi, la première équation d'Euler devient

$$
\frac{\mathrm{d} U}{\mathrm{~d} t}=-\frac{1}{\rho} \frac{\mathrm{d} p}{\mathrm{~d} x}=\left(\text { à très peu près) }-\frac{1}{\rho_{0}} \frac{\mathrm{d} p}{\mathrm{~d} x}\right.
$$

Faisons, d'autre part, dans l'équation usuelle $\frac{\mathrm{d} \cdot p^{5}}{\mathrm{~d} t}+$ $\frac{\mathrm{d} . \rho \mathcal{E} U}{\mathrm{~d} x}=\mathrm{o}$ de la conservation des masses, $\mathrm{p}=p_{0}\left(\mathrm{I}+\frac{p}{\tilde{k}}\right)$ et $\sigma=\sigma_{0}\left(\mathrm{I}+\frac{2 R}{E \dot{\varepsilon}} p\right)$, formules dont la première est évidinte et dont la seconde résulte de (1). Il viendra aisément, vu (2)

$$
\frac{\mathrm{d} u}{\mathrm{~d} x}=-\frac{\mathrm{I}}{P_{0} \omega^{2}} \frac{\mathrm{d} p}{\mathrm{~d} t}
$$

Or, l'élimination immédiate, soit de $U$, soit de $p$, entre (i) et (7) donne bien les deux équations cherchées.

\section{I}

Calcul, pour les diverses contextures et épaisseurs de paro: possibles, de la résistance élastique qu'un tujau sans lension longitudnale oppose au gonfliment de la colonne liquide le remplissant. $\left(^{*}\right)$

I. La particularité la plus délicate de la théorie des coups de bélier et, en général, des ondes liquides où intervient l'élasticité du tuyau contenant une colonne fluide en repos ou en mouvement, consiste dans lhypothese, inévjtable si l'on veut aboutrr, que les anneaux juxtaposés dont se compose le tuyau agissent, chacun pour son compte. sur le fluide intérieur, sans s'influencer mutuellement. Or cette hypothèse ne serait pleinement justifiée que pour un tuyau à fibres annulaires résistantes, mais dont les fibres longitudinales seraient, au contraire, infiniment extensibles et compressibles. Tel serait celui que donneraient, par exemple, lá superposition et la juxiaposition, en grand nombre, d'anneaux homogènes sans largeur ni épaisseur sensibles, où, encore, les enroulements multipliés d'un long fil élastiqué a spires très voisines, analogue aux trachées des végétaux, anneaux ou enroulements que relierait une sorte de parenchyme lâche, ou une toile affectée d'une double infinité de plis longttudinaux et transversaux. Il y a done quelque intérêt à attribuer au tuyau, conformément, d'ail-

(†) Séance du 10 juillet 1905. 
leurs, à la réalité, une contexture hétérotrope, différente suivant la longueur de ce qu'elle est dans les sens transversaux, de manière à pouvoir, du moins à la limite, le supposer, ainsi, infiniment extensible et compressible suivant sa longueur, ou composé effectivement d'anneaux contigus sans action appréciable les uns sur les autres.

Le but principal de cette Note sera, par conséquent, de trouver comment, dans un luyau élastique homogène, mais isotrope seulement autour de ses fibres longitudinales, et à surface extérieure censée libre de toute pression, le rayon $R$ se dilate lorsque croît la pression $p$ exercée sur sa face concave par le fluide contigu. La relation obtenue de la sorte rattachera la pression $p$ de ce fluide au rayon actuel de la section a qu'il occupe et sera précisément celle que la théorie de l'élasticité doit fournir à l'Hydrodynamique, pour déterminer le problème des mouvements du fluide. L'épaisseur primitive $\varepsilon$ du tube, différence de ses deux rayons, extérieur, $R_{1}$, et intérieur, $R$ à l'état naturel, sera d'ailleurs supposée avoir un rapport quelconque avec le rayon $R$ intérieur, et non plus en être une très petite fraction, comme je l'avais admis dans ma précédente Note (à la suite de Resal et de M. Alliévi), afin d'arriver au résultat le plus simple et d'y arriver le plus rapidement possible.

II. Le tuyau ayant comme axe d'isotropie de sa matière son axe même, choisi pour celui des $x$, il y aura lieu d'adopter, pour son potentiel $\Phi$ d'élasticité, qui exprime les six forces élastiques usuelles $N_{x}, N_{y}, N_{z}, T_{x}, T_{y}, T_{z}$ par ses six dérivées partielles premières relatives aux six déformations élémentaires bien connues $\partial_{x}, \partial_{y}, \partial_{z}, g_{x}, g_{y}, g_{\eta}$, la formule (6o), à cinq coefficients d'élasticité $\lambda, \mu, y, \lambda, \mu^{\prime}$, démontrée, à titre d'exercice, dans la septième de mes Leçons d'Analyse infinitésimale pour la Mécanique et la Phrsique (t. I, Compléments, p. I I9), savoir :

$$
\Phi=\left\{\begin{array}{l}
\frac{\lambda}{2}\left(\partial_{y}+\partial_{i}\right)^{2}+\lambda^{\prime} \partial_{x}\left(\partial_{y}+\partial_{i}\right)+\frac{\nu}{2} \partial_{x}^{2} \\
+\mu\left(\partial_{y}^{2}+\partial_{i}^{2}+\frac{1}{2} g_{x}^{2}\right)+\frac{\nu_{0}^{\prime}}{2}\left(g_{y}^{2}+g_{z}^{2}\right) .
\end{array}\right\}
$$

Elle donne pour les six forces $N, T$, en appelant $\theta$ la dilatation superficiolle $\partial_{y}+\partial_{i}$ des sections normales à l'axe d'isotropie ou des $x$,

$$
\begin{gathered}
N_{x}=\lambda^{\prime} \theta+\nu_{x}, \quad T_{x}=\mu g_{x}, \quad\left(T_{y}, T_{z}\right)=\mu^{\prime}\left(g_{y}, g_{z}\right), \\
\left(N_{y}, N_{z}\right)=\lambda_{\theta} \theta+\lambda^{\prime} \partial_{x}+2 \mu\left(\partial_{y}, \partial_{z}\right)
\end{gathered}
$$

On voit que les anneaux seront mutuellement indépendants, ou que la pression sur les sections normales du tuyau aura ses troits composantes $N_{x}, T_{z}, T_{y}$ essentiellement nulles, à la triple condition, nécessaire et suffisante, que $\lambda^{\prime}=0, \mu^{\circ}=0, \nu=0$; ce qui revient à réduire le potentiel $\Phi$, ou les six forces $N, T$, à leurs expressions ordinaires, en $\lambda$ et $\mu$, du cas d'isotropie, mais spécifiées pour des déformations planes, parallèles aux $y z$ et indépendantes des $x$, où l'on aurat $\partial_{x}=0, g_{\xi}=0, g_{y}=0$. Quant à l'isotropie complète on l'obtient en posant $\lambda^{\prime}=\lambda, \mu^{\prime}=\mu, \nu=\lambda+2 \mu$.

III. Mais ne nous bornons pas à ces deux cas; et admettons seulement la symétrie des déformations, tant par rapport aux sections normales que tout autour de l'axe des $x$. Cette dernière est évidente, par le fait même qu'on néglige
I

les poids soit du liquide, soit du tuyau, ainsique les appuis extérieurs de ce dernier, et à raison de la valeur commune attribuée à la pression intérieure $p$, sur tout le contour des sections normales du fluide. Quant à la symétrie des défor. mations du tuyau par rapport à ses propres sections normales, elle résulte, à très peu près, de la forme allongée qu'entraîne, pour les ondes, la rapidité de leur propagation. Par suite, ces sections resteront planes, normales à l'axe sensiblement rectiligne du tuyau et parallèles entre elles; en sorte que les fibres longitudinales, qui leur sont perpendin. culaires, éprouveront, toutes, la même dilatation (ou contraction) $\partial_{x}$, fonction seulement, comme $p$, de l'abscisse $x$ et du temps $t$.

Enfin, les considérations précédant la formule (I) de ma précédente Note font voir que chaque élément matériel de volume du tuyau peut être supposé, à tout instant, en équilibre, sous l'action des éléments contigus et, pour les plus voisins de l'axe, de la pression intérieure $p$.

IV. Cela posé, appelons, à la distance primitive $r$ de de l'axe, $\partial_{1}, \partial_{2}, \partial_{3}$ les trois dilatations linéaires principales de la matière du tuyau, savoir $: \partial_{4}$, la dilatation de la fibre, $d r$, prise suivant le prolongement du rayon $r$ émané perpendiculairement de l'axe jusqu'au point considéré; $\supset_{2}$, celle de la fibre annulaire $2 \pi r$ passant par ce point; enfin, $\partial_{3}$, la dilatation $\partial_{x}$, indépendante de $r$, de la fibre longitudinale émanée du même point. Si $\alpha$, fonction de $r$, désigne le petit déplacement de celui-ci dans sa section normale, allonge* ment élastique éprouvé par le rayon $r, \partial_{1}$ en sera la dérivée par rapport à $r$, et $\partial_{2}$, allongement relatif de la fibre circum laire $2 \pi r$ devenue $2 \pi(r+\alpha)$, vaudra le quotient de $\alpha$ par $r$. L'on aura donc, en observant que $\partial_{1}+\partial_{2}=0$.

$$
\mathrm{o}_{1}=\frac{\mathrm{d} \alpha}{\mathrm{d} r}, \quad \mathrm{o}_{2}=\frac{\alpha}{r}, \quad \theta=\frac{\mathrm{r}}{r} \frac{\mathrm{d}, r \alpha}{\mathrm{d} r}, \quad \mathrm{o}_{3}=\text { const. }
$$

et les formules (2) donneront pour les trois pressions (ou plutôt tractions) principales $P_{1}, P_{2}, P_{3}$ exercées sur les élé. ments plans normaux à $\partial_{1}, \partial_{2} \partial_{3}$,

$$
\left(P_{1}, P_{2}\right)=\lambda \theta+\lambda^{\prime} \partial_{3}+2 \mu_{1}\left(\partial_{1}, \partial_{2}\right), \quad P_{3}=\lambda^{\prime} \theta+\nu_{3} .
$$

Il en résulte notamment,

$$
\begin{gathered}
\frac{\mathrm{d} P_{1}}{\mathrm{~d} r}=\lambda \frac{\mathrm{d} \theta}{\mathrm{d} r}+2 \mu \frac{\mathrm{d}^{2} \alpha}{\mathrm{d} r^{2}} \\
P_{1}-P_{2}=2 \mu \cdot\left(\partial_{1}-\partial_{2}\right)=2 \mu \cdot r \frac{\mathrm{d}}{\mathrm{d} r}\left(\frac{\alpha}{r}\right) .
\end{gathered}
$$

Or, écrivons la condition d'équilibre, suivant le rayon $r$, d'un volume élémentaire (de longueur $\mathrm{I}$ dans le sens des $x$ ) compris entre les deux cylindres de rayons primitifs $r, r+\mathrm{d} r$ et deux plans menés suivant l'axe, inclinés res* pectivement de $\pm \frac{\gamma}{2}$ par rapport au rayon $r$. Sur ses deux faces courbes $\gamma r, \gamma(r+\mathrm{d} r)$, les tractions exercées seront, suivant le rayon $r,-\gamma r P_{1}, \gamma\left(r P_{1}+\mathrm{d} r P_{1}\right)$; et leur résultante algébrique:

$$
\gamma \mathrm{d}\left(r P_{1}\right) \text { ou } \gamma r \mathrm{~d} P_{1}+\gamma P_{1} \mathrm{~d} r
$$

se trouvera équilibrée par la projection, sous l'angle 
$\frac{5}{2}-\frac{\gamma}{2}$, des deux tractions normales $P_{2} \mathrm{~d} r$ exercées sur les deux faces planes latérales $\mathrm{d} r$. L'on aura donc

$$
\gamma r \mathrm{~d} P_{1}+\gamma P_{1} \mathrm{~d} r=\gamma P_{2} \mathrm{~d} r
$$

c'est-à-dire, en simplifiant et utilisant finalement les formules (5), (3) ci-dessus,

$$
\begin{aligned}
& \lambda \frac{\mathrm{d} \theta}{\mathrm{d} r}+2 \mu \frac{\mathrm{d}}{\mathrm{d} r}\left(\frac{\mathrm{d} \alpha}{\mathrm{d} r}+\frac{\alpha}{r}\right)=0 \\
& \text { ou } \quad(\lambda+2 \mu) \frac{\mathrm{d} \theta}{\mathrm{d} r}=0 .
\end{aligned}
$$

V. La dilatation superficielle $\theta$ des sections normales du tuyau est donc constante, tout comme $\partial_{3}$; et la formule (4) de $P_{3}$ montre que l'action mutuelle de deux anneaux contigus se trouve répartie uniformément sur leur base commune. Son annulation admise oblige donc à poser $P_{3}=0$; et il vient successivement, vu (4) et (3) en appelant $A$ une constante arbitraire.

$$
\partial_{3}=-\frac{\lambda^{\prime}}{\gamma} \theta, \quad \alpha=\frac{\theta}{2} r+\frac{A}{r}, \quad P_{1}=\frac{(\lambda+\mu) y-\lambda^{\prime 2}}{y} \theta-\frac{2 \mu \cdot A}{r^{2}}
$$

La constante $A$ se détermine par la condition que $P_{1}$ s'annule à la face externe $r=R_{1}$ (supposée libre du tuyau).

Enfin, la valeur $p$, de $-P_{1}$ pour $r=R$, c'est-à-dire à la face interne, et celle, $\partial^{\prime}$, de la dilatation $\partial_{2}$ à la même limite $r=R$, seront

$$
\left.\begin{array}{l}
p=\frac{(\lambda+\mu) \nu-\lambda^{\prime 2}}{y} \frac{R_{1}^{2}-R^{2}}{R^{2}} \theta, \\
\partial^{\prime}=\frac{(\lambda+2 \mu) \nu-\lambda^{\prime 2}}{2 \mu . y}\left[\mathrm{I}+\frac{(\lambda+\mu) \nu-\lambda^{\prime 2}}{(\lambda+2 \mu) \nu-\lambda^{\prime 2}} \frac{R_{1}^{2}-R^{2}}{R^{2}}\right] \theta .
\end{array}\right\}
$$

Si l'on observe que le coefficient $E$ d'élasticité des fibres annulaires, rapport de $P_{2} \grave{a} \partial_{2}$ quand $P_{1}$ et $P_{3}$ sont nuls, à la valeur $4 \frac{(\lambda+\mu) y-\lambda^{\prime 2}}{(\lambda+2 \mu) y-\lambda^{\prime 2}} \mu$, ces deux relations $(7)$ conduiront, pour le quotient de $p$ par a' à la formule simple

$$
\begin{gathered}
\text { où } \quad E^{\prime}=E \frac{\frac{p}{\partial^{\prime}}=E^{\prime} \stackrel{\varepsilon}{R},}{1+4 \frac{(\lambda+\mu) y-\lambda^{\prime 2}}{(\lambda+2 \mu) \nu-\lambda^{\prime 2}}\left(1+\frac{\varepsilon}{2 R}\right) \frac{\varepsilon}{2 R}}
\end{gathered}
$$

Cette formule ne diffère de celle, (I), de ma précédente Note et, par suite, la vitesse $\omega$ de propagation des ondes ne sedistinguera des célérités obtenues par Résal et $\mathrm{M}$. Alliévi, qu'en ce que le coefficient usuel $E$ ' d'élasticité des fibres annulaires s'y trouve remplacé par un autre, $E^{\prime}$, non moins constant pour chaque tuyau, et dont le rapport à $E$, dépendant à la fois de la contexture du tuyau et du rapport même de son épaisseur $\varepsilon \dot{a}$ son diamètre $2 R$, tend vers l'unité quand ce dernier rapport tend vers zéro, tout en étant généralement plus petit que $\mathrm{I}$. Si l'on considère, en effet, le cas moyen d'une contexture isotrope (où l'on a $\lambda^{\prime}=\lambda$, $v=\lambda+2 \mu$ ) et le cas extrême d'une contexture assurant lindépendance mutuelle des anneaux (où $\lambda$, y sont infiniment petits du même ordre), il vient aisément, en appelant $K, K_{1}$ les deux valeurs correspondantes de $\frac{E}{E}$,

$$
\begin{aligned}
\left(1+\frac{\varepsilon}{2 R}\right)(K-\mathrm{I}) & =\frac{\varepsilon}{2 R}\left(\frac{2 \lambda+\mu}{\lambda+\mu}+\frac{3 \lambda+2 \mu}{\lambda+\mu} \frac{\varepsilon}{2 R}\right), \\
K_{1}-K & =\frac{\lambda}{\lambda+\mu} \frac{\lambda}{\lambda+2 \mu} \frac{\varepsilon}{2 R},
\end{aligned}
$$

et, par conséquent, $K>\mathrm{r}, K_{1}>K$. Le rapport inverse de $E$ 'à $E$ est donc inférieur à l'unité, surtout dans le second cas.

VI. Ces raisonnements précédents ne s'appliquent guère, il est vrai, à une conduite d'eau, non seulement rigide, mais enfoncée dans un terrain qui s'oppose à ses mouvements un peu étendus. Néanmoins, admettons que les ondes s'y propageant n'aient qu'une longueur restreinte, ou, si elles sont longues, que l'excès de pression dû à leur passage soit de signes variés, et nul en moyenne sur sur des longueurs modérées. Comme, dans chaque section, cet excès donne sur toute la paroi concave d'un anneau des efforts ayant, suivant tout axe coordonné, composante totale et moment total nuls, l'effet de ces efforts pour déformer et entraîner le tuyau ne peut être que très local, c'est-à-dire devenir insignifiant à toute distance comprenant un nombre suffisant de fois le rayon $R$; car il suffirait, en les transportant sur l'axe (supposé alors relié à la paroi), de les déplacer d'une quantité égale à $R$, négligeable par conséquent à côté de la distance dont il s'agit, pour les faire annihiler les uns par les autres.

Donc les déformations du tuyau ne seront appréciables, si l'onde est courte, que dans son voisinage: elles ne tendront alors, nulle part, à produire, par leur accumulation, des déplacements sensibles. Et si, au contraire, l'onde est indéfinie, mais composée de parties de longíeur restreinte, donnant lieu à des excès de pression moyennement nuls, on conçoit que les déformations d'un tronçon du tuyau dues à 1a totalité des parties de l'onde éloignées, seront encore négligeables, en raison de la neutralisation mutuelle, qui s'y produira, des effets d'efforts positifs et d'efforts négatifs exercés assez près les uns des autres.

Le tuyau ne tendant ainsi à éprouver que des déformations, ou trés localisées, ou de signes contraires sur des tronçons assez voisins, il n'en résultera nulle part, comme on voit, des déplacements notables, susceptibles, par suite, d'être très gênés par la résistance du terrain ou même par celle des extrémités (si l'on en est assez loin); et l'hypothèse de l'indépendance mutuelle d'anneaux contigus à surface extérieure libre devra donner encore, par les formules (8) qu'elle entraîne, une approximation acceptable.

\section{III}

Sur un cas simple, où se calculent aisément l'action mutuelle des anneaux juxtaposés constituant un turau et l'influence de cette action mutuclle sur la propagation des ondes liquides dans le turau. (*)

I. J'ai donné dans deux notes récentes, à la suite de Résal et de $M$. Alliévi, les équations régissant la propagation du mouvement le long de la colonne liquide contenue dans un tuyau élastique sans tension longitudinale, mais en

(*) Séance du 24 juillet I 905 . 
observant qu'une tension de ce tuyau suffisante pour modifier la dilatation des anneaux sous l'action du fluide inclus compliquerart beaucoup le problème. Voici un cas simple où la question reste néanmoins résoluble.

II. C'est le cas d'un tuyau droit sans pesanteur, maintenu seulement par ses deux extrémités, et que les considérations de la fin du no II de ma première note permettraient de supposer sans cesse à l'état d'équilibre intérieur, mềne dans son ensemble. La pression totale entre anneaux contigus, dès lors constante d'une extrémité à l'autre (*) et égale à la poussée sur les apruis, pourra être censée proportionnelle aux déplacements de ceux-ci et, par conséquent, à leur somme, c'est-à-dire à l'allongement même de tout le tuyau, allongement que j'appellerai $\Delta$, et qui sera l'intégrale $\int \partial_{3} \mathrm{~d} x$ avec les notations de ma deuxième note, aux calculs de laquelle je prierai le lecteur de se reporter.

Mais la tension $P_{3}$ entre anneaux, par unité d'aire, vu sa formule (4) linéaire en $\partial_{3}$ et $\theta$, sera indépendante de $r$ comme $a_{3}$ et 0 , indépendante aussi, par suite, de $x$ comme sa valeur moyenne, et, en conséquence, proportionnelle à $\Delta, \mathrm{Or}$, de là, résultera une mise en compte, bien simple, de cette action mutuelle des anneaux.

En effet, l'expression de $\partial_{3}$ donnée au no $\mathrm{V}$ (note 11 ), puis celles (7) de $p$ et de $\partial$ ', ne s'accroitront, chacune, que d'un terme encore profortionnel à $P_{3}$ ou à $\Delta$; et l'élimination de 0 , entre les deux relations (7) ainsi complétées, n'ajoutera, de mêmé, à l'expression linéaire de $\partial$ ' en $p$, qu'un terme proportionnel à $\lrcorner$. Par suite, la formule $\sigma_{0}\left(1+20^{\prime}\right)$ de la isection fluide $\sigma$ s'arcroissant d'un terme analogue, celle du produit $p \sigma$, considéré vers la fin de ma première note pour obtenir l'équation (7) de cette note, prendra la forme

$$
\hat{p}_{0} \sigma_{0}\left(1+\frac{p+a \Delta}{p_{0} \omega^{2}}\right)
$$

si l'on y appelle $a$ un coefficient constant connu. Il suffira donc de poser

$$
p+a \Delta=p^{\prime},
$$

poụr obtenir, en $p^{\prime}$ et $U$, les équations (6), (7) et, par suite (5) de la même note, avec $p$ simplement accentué.

D'ailleurs, l'expression de $\partial_{3}$ fournie par la substitution, dans la formule de $\partial_{3}$ déjà obtenue, à 0 , de sa valeur linéaire en $p$ et $\Delta$, réduit finalement la relation $\Delta=\int \partial_{3} \mathrm{~d} x$ à deux termes, l'un, en $\Delta$, l'autre, en $\int p \mathrm{~d} x$; et, donnant ainsi $\Delta$ proportionnel à $\int p \mathrm{~d} x$, elle permet de déduire à toute époque $t$ (notamment à l'instant initial $t=0$ ), les valeurs de $p$ de celles de $p$, ou vice versa.

Par conséquent, sauf cette substitution de $p$ 'à $p$, les lois du mouvement seront les mêmes que dans un tuyau sans tension longitudinale, et la vitsse $\omega$ de propagation des ondes ne dépendra pas du degré de fixité des deux sections extrêmes.

(') Cette constance n'est pas compromise, dans le cas d'une épaisseur E seulement comparable aux petites variations du rayon intérieur, par la composante, suivant l'axe, des pressions $p$ exercées sur la face concave, alors négligeables devant la tension Ej' des anneaux.
III. En résumé, grâce à l'hypothèse d'inerties négligea. bles du tuyau considéré même dans sa totalité, l'influence que l'action mutuelle des anneaux contigus rend possible, sur chaque tronçon de la colonne fluide, aux tronçons éloignés, s'exprime par le terme inlégral unique $a \Delta$, propor. tionnel ̀̀ $\int p \mathrm{~d} x$, et dont l'adjonction à la pression $p$ permet à celle-ci, changée ainsi, fictirement, en $p$, de jouer le même rôle que si le tuyau était un tube lâche $\left(^{*}\right)$.

\section{LE MOIS HYDRO-ÉLECTRIQUE}

\section{ACADÉMIE DES SCIENCES}

\section{MÉGANIQUE ET ÉLECTRICITÉ}

Sur les phénomènes de l'arc chantant. - Note de $M . A_{\text {; }}$ Blondel, séance du 26 juin 1905.

Jai reconnu, par l'analyse d'un grand nombre de courbes oscillographique $\left({ }^{\star \star}\right)$, qu'avec les électrodes en charbon homogène, on peut ohtenir des types bien différents d'arcs chantants, l'un contunu, lautre discontin' dont les courbes sont bien caracterisées respectivement par les figures i et 2 ci-jorntes, obtenus tous les deux avec la tension d'alimentation crdinaire, voisine de 120 volts et une fable self-induction dans le circuit d'oscillation.

Le premier typo, figure I, auquel correspond un son musical assez pur, se présente seulement si l'écart entre les charbons est plutôt fort et au moins de l'ordre de celui dun bon arc ind 1 striel $(3 \mathrm{~m} / \mathrm{m} 5 \mathrm{a}$ $4 \mathrm{~m} / \mathrm{m}$ ou davantage) et, le plus facilement quand le circuit d'alimentation $\mathrm{ABDE}$ ne contient qu'une résistance morte ou fablemenl inducive et que le courant n'est pas trop voisin du courant limite de stabilité.

Le detuxième type, figure 2, auquel correspond un son plus stridem ou sifflant, s'obtient quand l'are est court par rapport au voltagt employé (par exemple o m/m 5 à $\mathrm{Im} / \mathrm{m} 5$ à la tension de 110 :
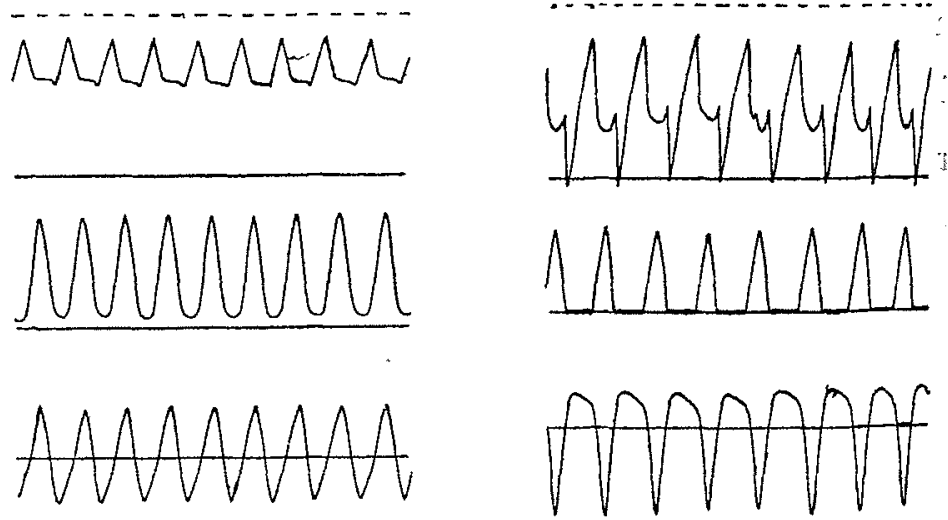

Fig. 1.

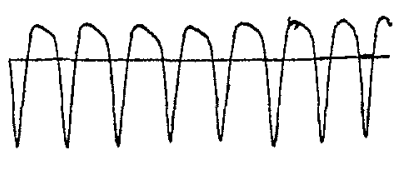

Fig. 2.

Fig. 1 . Ecart: $4 \mathrm{~m} / \mathrm{m}$; intensité : 2,2 ampères; tension: 126 voits (self: $L=0$ ); $C=16$ microfarads et $l=0,00345$ henry; frequenct mesuree 540. - Courbe superieure: tension entre les bornes de larc l'horizontale superieure représente la tension de la source. - Courbi intermediaire : courant dans l'arc. - Courbe inférieure : courant dí condensateur.

Fig. 2 Ecart $\mathrm{Im} / \mathrm{m}^{\prime} 3$; intensité moyenne 14 ampères $(L=0290) C=1 t$ microfarads et $l=0,0034$ henry; fréquence mesurée 457 .

( $\left.{ }^{*}\right)$ Je viens d'apprendre que l'expression binome (2) de M. Alliéví pour l'inverse du carré $\omega^{2}$ de la célérité, avait été donnée dès 1878 par M. Dorteweg, dans les Annalen der Physik und Chemie, $t, V$, p. 525 à 542 .

(*) J'ai fat construire, dans ce but, en 5903, par MM. Dobkevitch e Nagel,un oscillographe triple bipolaire (d'apres le principe que fai expost à l'dcademie.en mars ı go '). J'at eté assiste pour l'exécution des expériencé (executées de janvier à avril r go5), par MM. Boutin et Bethenod, ingénieưfí électriciens, qui mont preté le concours le plus efficace. 